\title{
Helioseismology of sunspots: defocusing, folding, and healing of wavefronts
}

\author{
Z.-C. Liang ${ }^{1,2}$, L. Gizon ${ }^{2,3}$, H. Schunker ${ }^{2}$, and T. Philippe ${ }^{4,2}$
}

\author{
1 Department of Physics, National Tsing Hua University, Hsinchu 30013, Taiwan (R.O.C.) \\ 2 Max-Planck-Institut für Sonnensystemforschung, 37191 Katlenburg-Lindau, Germany \\ e-mail: gizon@mps .mpg. de \\ 3 Institut für Astrophysik, Georg-August-Universität Göttingen, 37077 Göttingen, Germany \\ ${ }^{4}$ Université de Toulouse, ISAE-SUPAERO, 10 avenue Edouard Belin, 31055 Toulouse Cedex 4, France
}

Received 15 March 2013 / Accepted 21 June 2013

\begin{abstract}
We observe and characterize the scattering of acoustic wave packets by a sunspot in a regime where the wavelength is comparable to the size of the sunspot. Spatial maps of wave travel times and amplitudes are measured from the cross-covariance function of the random wave field observed by SOHO/MDI around the sunspot in active region NOAO 9787. We consider separately incoming plane wave packets consisting of $\mathrm{f}$ modes and $\mathrm{p}$ modes with radial orders up to four. Observations show that the travel-time perturbations diminish with distance far away from the sunspot - a finite-wavelength phenomenon known as wavefront healing in scattering theory. Observations also show a reduction of the amplitude of the waves after their passage through the sunspot. We suggest that a significant fraction of this amplitude reduction is due to the defocusing of wave energy by the fast wave-speed perturbation introduced by the sunspot. This "geometrical attenuation" will contribute to the wave amplitude reduction in addition to the physical absorption of waves by sunspots. We also observe an enhancement of wave amplitude away from the central path: diffracted rays intersect with unperturbed rays (caustics) and wavefronts fold and triplicate. Wave amplitude measurements in time-distance helioseismology provide independent information that can be used in concert with travel-time measurements.
\end{abstract}

Key words. Sun: helioseismology - sunspots - Sun: activity - Sun: oscillations - Sun: interior - Sun: surface magnetism

\section{Introduction}

The propagation of solar seismic waves is affected by sunspots. The scattering phase shifts and wave absorption coefficients can be measured by several techniques, such as Fourier-Hankel analysis and time-distance analysis (see review by Gizon et al. 2010, and references therein).

Cameron et al. (2008) studied the interaction of $f$-mode plane-wave packets with a sunspot using time-distance helioseismology. As we describe briefly below, the data-averaging strategy used by Cameron et al. (2008) reduces the noise and enables a detailed study of the waveforms far from the scattering region. In this study, we extend the observations to modes with different radial orders and wavelengths that are closer to the sunspot radius, in order to investigate finite-wavelength effects (e.g. Nolet \& Dahlen 2000; Hung et al. 2001). We measure not only traveltime shifts but also wave amplitude perturbations with respect to the quiet Sun.

We use one-minute cadence Doppler velocity images measured by the Michelson Doppler Imager (Scherrer et al. 1995) onboard the Solar and Heliospheric Observatory (SOHO/MDI). The images are remapped using Postel's azimuthal equidistant projection in order to track the motion of the sunspot in Active Region 9787 (Cameron et al. 2008; Gizon et al. 2009; Moradi et al. 2010). The map scale is 0.12 heliographic degrees per pixel. Nine data sets are obtained, one for each day of the period 20-28 January 2002. The selected sunspot is nearly circular
Table 1. Characteristics of incoming wave packets.

\begin{tabular}{lccrrr}
\hline \hline Modes & $n$ & $\begin{array}{c}v \\
(\mathrm{mHz})\end{array}$ & $\begin{array}{c}\lambda \\
(\mathrm{Mm})\end{array}$ & $R / \lambda$ & $\begin{array}{c}x_{\mathrm{F}} \\
(\mathrm{Mm})\end{array}$ \\
\hline$f$ & 0 & 2.6 & 6.3 & 3.2 & 126.2 \\
$p_{1}$ & 1 & 2.9 & 8.3 & 2.4 & 95.3 \\
$p_{2}$ & 2 & 3.1 & 10.7 & 1.9 & 73.4 \\
$p_{3}$ & 3 & 3.4 & 13.1 & 1.5 & 59.4 \\
$p_{4}$ & 4 & 3.6 & 14.8 & 1.4 & 52.2 \\
\hline
\end{tabular}

Notes. A wave packet consists of waves with the same radial order $n$ (ridge filtering). The quantity $x_{\mathrm{F}}$ is the distance from the sunspot such that the sunspot fills the first Fresnel zone of the scattered wave packet. See Fig. 2 and Sect. 4.

and does not evolve significantly over the duration of the observations, $T=9$ days. Its outer-penumbral radius is $R=20 \mathrm{Mm}$.

Filters are applied to the Dopplergrams in 3D Fourier space to extract waves with the same radial order (ridge filters). Five different filters are applied, for modes $f$ through $p_{4}$ (corresponding to radial orders $0 \leq n \leq 4)$. Table 1 lists the characteristics of the wave packets for each radial order. The mean frequency, $v$, and mean wavelength, $\lambda$, of each wave packet are defined as weighted averages over the filtered power spectrum: $\lambda$ increases with $n$, as does $v$ due to the filtering. The finite-wavelength effects of scattering by the sunspot are expected to be important in the regime where $R / \lambda \lesssim 1$, a condition that is discussed further 
in Sect. 4. These effects should thus be easier to detect with increasing radial order $n$.

\section{Cross-covariance function and wave packets}

The cross-covariance of the filtered Doppler velocity is computed in the same way as in Cameron et al. (2008):

$C_{n}(x, y, t)=\int_{0}^{T} \bar{\phi}_{n}\left(t^{\prime}\right) \phi_{n}\left(x, y, t^{\prime}+t\right) \mathrm{d} t^{\prime}$,

where $(x, y)$ is a local Cartesian coordinate system with its origin at the center of the sunspot, $t$ is the time, $\phi_{n}$ the filtered Doppler velocity for modes with radial order $n$, and $\bar{\phi}_{n}$ the average of $\phi_{n}$ over the line $L$ at $x=-43.73 \mathrm{Mm}$. The spatial averaging over $L$ is equivalent to filtering out all the wavevectors that are not perpendicular to $L$ : only waves that propagate toward and away from $L$ contribute to $\bar{\phi}_{n}$. In addition, to reduce noise, the crosscovariances are averaged over $T=9$ days and over rotations about the sunspot center, as in Cameron et al. (2008) and Moradi et al. (2010).

As explained by Gizon et al. (2010), for $t>0$, the crosscovariance is used to follow the propagation of two plane wave packets initially at $L$ and propagating toward $+x$ and $-x$ respectively. The forward scattering of the waves by the sunspot occurs in the $(x>0, t>0)$ quadrant.

\section{Wave travel times and amplitudes}

We define the travel-time shift, $\Delta \tau(x, y)$, as the time lag that maximizes the similarity between the measured cross-covariance, $C_{n}(x, y, t)$, and a sliding quiet-Sun cross-covariance, $C_{n}^{\mathrm{qs}}(x, t)$. More precisely, $\Delta \tau$ is the time $\tau$ that maximizes the function

$F_{n}(x, y, \tau)=\frac{\int C_{n}(x, y, t) C_{n}^{\mathrm{qs}}(x, t-\tau) \mathrm{d} t}{\int\left|C_{n}^{\mathrm{qs}}(x, t)\right|^{2} \mathrm{~d} t}$

The quiet-Sun cross-covariance $C_{n}^{\mathrm{qs}}(x, t)$ is constructed by averaging $C_{n}(x, y, t)$ over $|y|>100 \mathrm{Mm}$, far away from the region of wave scattering, and it is zeroed out for $t<0$. The cross-correlation travel-time shift defined here is also used in geophysics (e.g. Zaroli et al. 2010) and is analogous to the definition of Gizon \& Birch (2002). The cross-correlation $F_{n}(x, y, \tau)$ at each spatial point $(x, y)$ as a function of $\tau$ is demodulated using the Hilbert transform to obtain its envelope. The maximum, $A(x, y)$, of the envelope of $F_{n}(x, y, \tau)$ is a measure of the wave packet amplitude. By definition, $A$ is equal to one in the quiet Sun. An example measurement of $\Delta \tau$ and $A$ for $C_{4}$ at $(x, y)=(60,0) \mathrm{Mm}$ (after interaction with the sunspot) is shown in Fig. 1.

For each wave packet, we obtain spatial maps of the traveltime shift $\Delta \tau(x, y)$ and wave amplitude $A(x, y)$. Figure 2 shows the maps of $\Delta \tau(x, y)$ and $A(x, y)$ for modes $p_{1}$ through $p_{4}$. The maps of $\Delta \tau$ clearly show negative travel-time anomalies behind the sunspot along $y=0$ as a result of the increase in the wave speed in the sunspot (Cameron et al. 2008), while the maps of $A$ show the amplitude reductions.

We only display the results for distances for $L$ larger than three wavelengths in each panel in order to exclude the near field where the $t<0$ and $t>0$ branches of $C_{n}$ are not well separated. This is not a limitation of this work since we are interested in wave scattering in the far field.

For $\mathrm{p}_{1}$ modes, it is interesting to note the positive travel-time anomalies on the left side of the penumbra $(x<0)$, caused by the

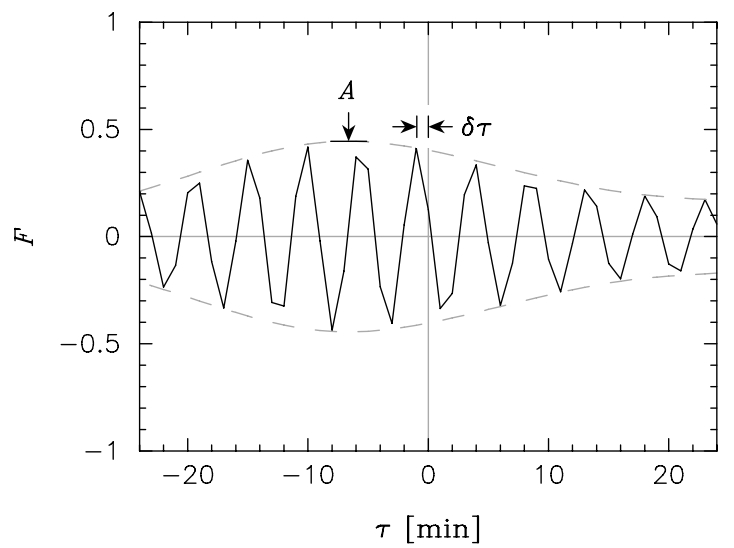

Fig. 1. Cross-correlation function $F_{4}(x, y, \tau)$ (solid line) between the cross-covariance $C_{4}(x, y, t)$ at $(x, y)=(60,0) \mathrm{Mm}$ and the corresponding quiet-Sun cross-covariance $C_{4}^{\mathrm{qs}}$. The envelope (dashed line) is determined by demodulation. The travel-time shift, $\Delta \tau$, and the wave packet amplitude, $A$, are indicated by arrows. In practice, we fit the peak nearest to $\tau=0$ with a parabola to measure the time shift. In this particular example, the time shift and reduced amplitude are $\Delta \tau=-57 \mathrm{~s}$ and $A=0.44$ with respect to the quiet Sun.

outward-directed flows in the penumbra and the moat. The effect of the moat flow is not seen in the far field: waves propagate against the moat flow for $x<0$ and with the moat flow for $x>0$.

\section{Wavefront healing and finite-wavelength effects}

The lefthand panel of Fig. 3 shows the travel-time shifts as a function of $x$ along $y=0$. For all the modes, the magnitude of the travel-time shift gradually decreases as waves propagate away from the sunspot. This phenomenon, called wavefront healing (Nolet \& Dahlen 2000; Hung et al. 2001), is inevitable because the amplitude of the scattered wave (a "circular" wave) decreases with distance from the sunspot more rapidly than the amplitude of the direct wave (a plain wave) by a factor proportional to the square root of distance. Wavefront healing is a finite-wavelength effect (Nolet \& Dahlen 2000). In particular, single-ray theory cannot explain wavefront healing but instead gives a constant travel-time perturbation for $x>R$ (Gizon et al. 2006).

The occurrence of finite-wavelength effects at a measurement point depends on whether the diameter of the sunspot is smaller than the width of the first Fresnel zone of that point. For any observation point $(x, y)$ that enters the computation of the cross-correlation (Eq. (1)), the corresponding first planewave Fresnel zone is bounded by a parabola with focus at this point (Gudmundsson 1996). Assuming a path length perturbation of $\lambda / 4$, the width of this Fresnel zone measured along $x=0$ is given by $\Delta y_{\mathrm{F}}(x)=2 \sqrt{(x+\lambda / 4)^{2}-x^{2}}$ (Gudmundsson 1996). We define the characteristic observation point $\left(x_{\mathrm{F}}, 0\right)$ such that the sunspot fills the first Fresnel-zone width, i.e. $\Delta y_{\mathrm{F}}\left(x_{\mathrm{F}}\right)=2 R$. For each wave packet, the value of $x_{\mathrm{F}}$ is provided in Table 1 and the corresponding Fresnel zone is the white curve in the top panels of Fig. 2. In the regime $x>x_{\mathrm{F}}$, the first Fresnel zone is always larger than the sunspot: finite-wavelength effects dominate and the ray approximation is no longer valid.

The righthand panel of Fig. 3 shows $p_{4}$-mode travel-time shifts as a function of $y$, at four different values of $x$. As the distance away from the sunspot increases, we see wavefront healing around $y=0$. In addition, we see that the width of the traveltime perturbation in the transverse direction grows with $x$. This observation cannot be explained in the context of linearized ray 

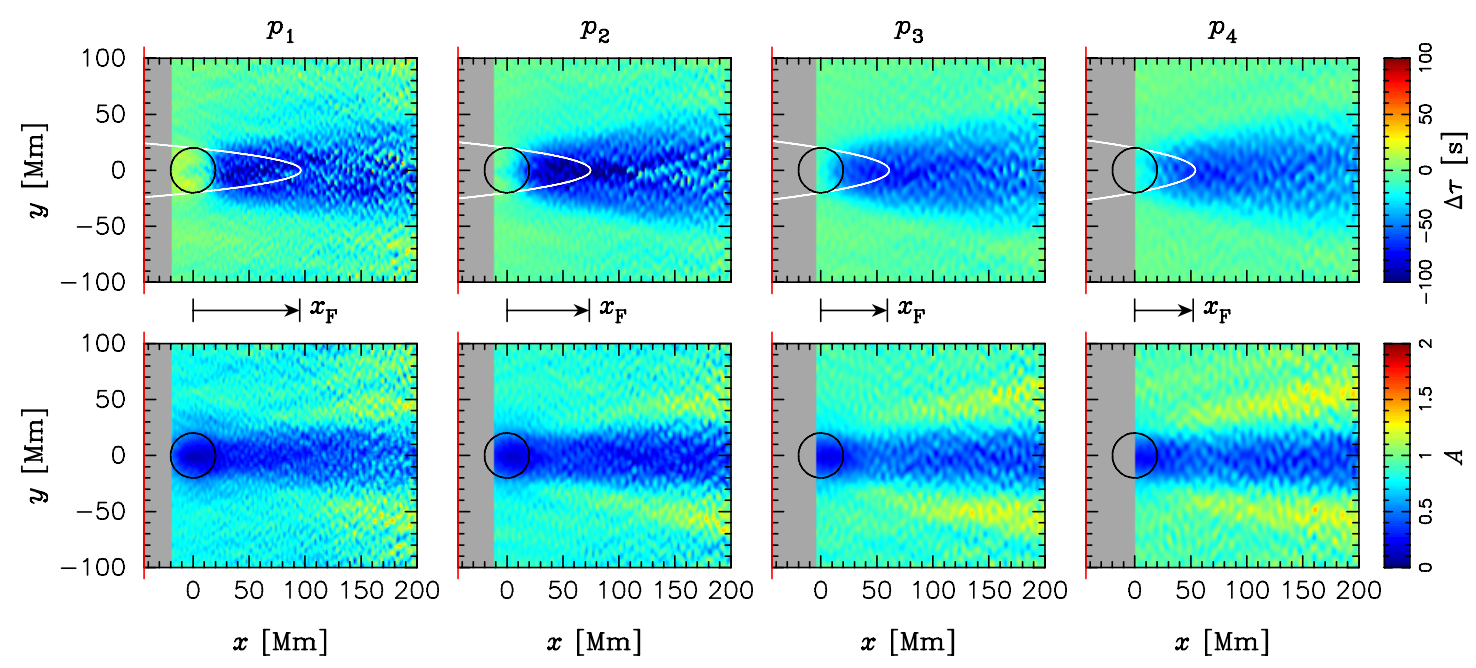

Fig. 2. Spatial maps of travel-time shifts $\Delta \tau(x, y)$ (top) and wave packet amplitudes $A(x, y)$ (bottom) with respect to the quiet Sun. From left to right are results for wave packets $p_{1}, p_{2}, p_{3}$, and $p_{4}$. Waves propagate from $L$ (red lines) in the $+x$ direction. The black circles denote the outer boundary of the penumbra of the sunspot. The parabolic curves (white lines with equation $\left.(y / R)^{2}+x /\left(x_{\mathrm{F}}+\lambda / 8\right)=1\right)$ depict the boundaries of the first plane-wave Fresnel zones with focus at $(x, y)=\left(x_{\mathrm{F}}, 0\right)$, chosen such that the sunspot fills the width of the Fresnel zone. The focus position $x_{\mathrm{F}}$ depends on radial order and is taken from Table 1. We do not display the results in the near fields (gray regions where distances from $L$ are less than $3 \lambda)$.
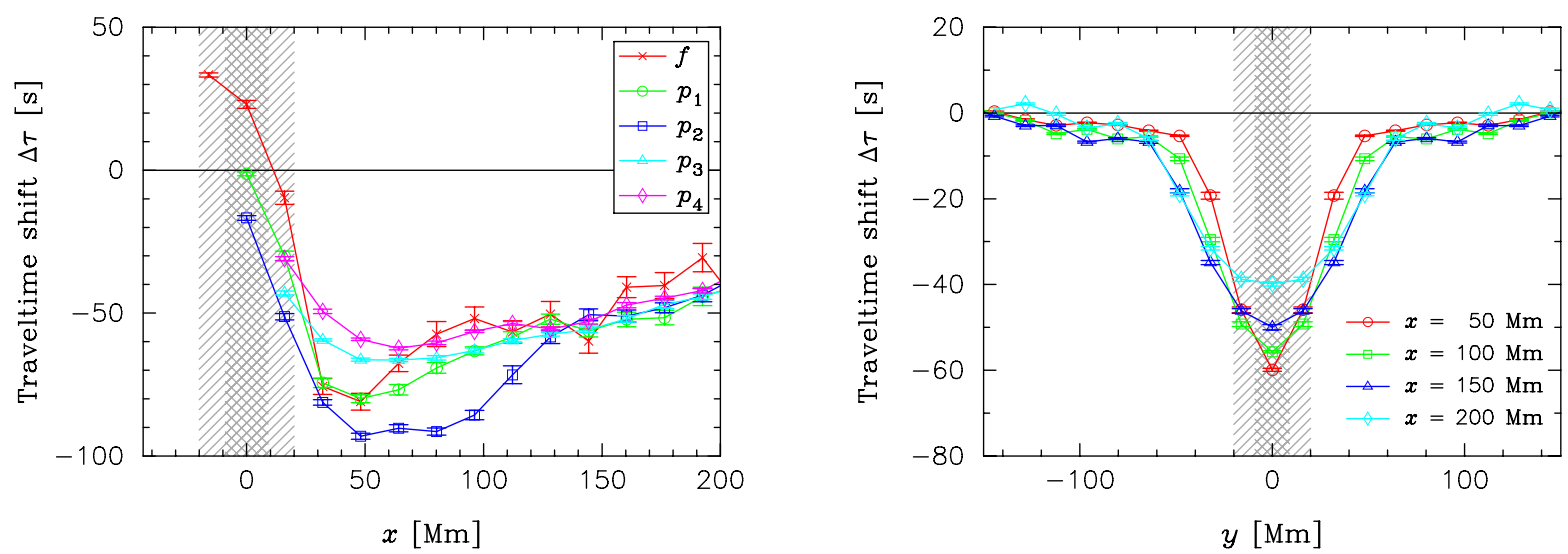

Fig. 3. Observed travel-time shifts, $\Delta \tau$. The left panel shows the travel-time shifts along $y=0$ for modes $f$ through $p_{4}$, which heal as the distance from the sunspot, $x$, increases. The right panel shows the travel-time shifts of $p_{4}$ modes as functions of $y$ at four different values of $x$. Notice the spread in the transverse direction as $x$ increases. The hatched and cross-hatched area indicate the locations of the penumbra and umbra, respectively. The data is averaged over patches of size $16 \times 16 \mathrm{Mm}^{2}$ to filter out spatial scales that are dominated by noise. Error bars give the standard deviation of the mean.

theory, according to which travel-time perturbations are computed along unperturbed paths $(y=$ const.) by using Fermat's principle (Hung et al. 2001). This is another warning against using linearized ray theory in sunspot seismology.

As we see in the next section, a simple ray-tracing calculation (geometrical optics) indicates that a fast wave-speed anomaly, like a sunspot, would defocus the rays and lead to travel-time perturbations that spread in the transverse direction as $x$ increases. However, ray tracing will still overestimate the travel-time shifts in the far field and would be incapable of explaining wavefront healing along $y=0$.

\section{Amplitude enhancements and caustics}

The bottom panels of Fig. 2 show that the transmitted wave packets have a reduced amplitude around $y=0$ compared to the quiet-Sun value, for all radial orders. It is known that wave absorption by sunspots causes a reduction in outgoing wave amplitude (Braun et al. 1987, 1988), as the result of partial mode conversion of incoming waves into slow magneto-acoustic waves that propagate down the sunspot (e.g. Spruit \& Bogdan 1992; Cally \& Bogdan 1997).

In our case (plane wave geometry), a significant fraction of the amplitude reduction that is observed on-axis behind the sunspot is due to the defocusing of wave energy by the fast wave-speed perturbation introduced by the sunspot. This can be illustrated by a simple $2 \mathrm{D}$ ray tracing experiment shown in the lefthand panel of Fig. 4, where the sunspot is approximated by a $10 \%$ enhancement of the wave speed. This "geometrical attenuation" will contribute to the wave amplitude reduction in addition to the physical absorption of waves. Their respective contributions would require better modeling since ray tracing cannot take wavefront healing into account.

Apart from the on-axis amplitude reduction, an enhancement of wave amplitude is seen away from the axis $y=0$ for modes $p_{2}, p_{3}$, and $p_{4}$ (bottom panels in Fig. 2). This off-path enhancement is more pronounced for the $p_{4}$ wave packet (right panel of Fig. 4). As above, we suggest that this off-path feature 

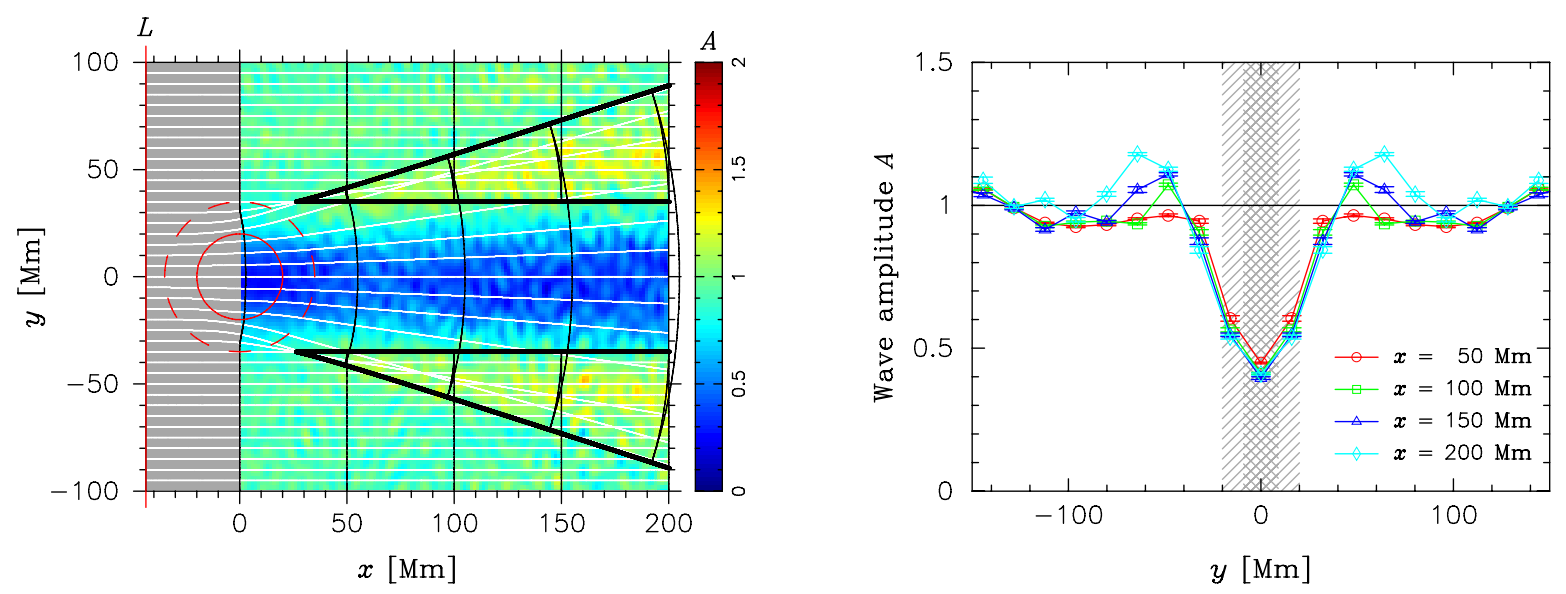

Fig. 4. Wave amplitude perturbations for $p_{4}$ caused by the sunspot. Left: map of observed amplitudes $A$ (color scale). The white lines are 2D rays traced through a sunspot model, such that the wave speed is enhanced by $10 \%$ inside the circle and transitions smoothly to the background value $\left(53.28 \mathrm{~km} \mathrm{~s}^{-1}\right)$ at the dashed circle. The wavefronts are given by the black lines. The thick black lines indicate the boundaries of the caustics where rays intersect and wavefronts fold. Right: observed amplitudes $A$ as a function of $y$ at fixed distances from the sunspot ranging from $x=50$ to $200 \mathrm{Mm}$. Notice the amplitude enhancements around $y \sim \pm 60 \mathrm{Mm}$.

is caused by sunspot-induced refraction. Since rays tend to bend away from the higher-speed medium (the sunspot), diffracted rays will cross over the unperturbed rays on both sides off the central axis. The envelope of intersecting rays is called a caustic in optics. The higher density of rays inside the caustics explains the enhancement of wave amplitude. The wavefronts fold and triplicate as they pass through the caustics (e.g. Dahlen \& Tromp 1998). Inside the caustics, multiple arrivals contribute to the cross-correlation travel-time measurements and the interpretation of travel time becomes ambiguous under the raytheoretical picture (Nolet \& Dahlen 2000; Hung et al. 2001). The location of the caustics depends on the perturbation strength and the sunspot geometry. In the case of $p_{4}$ modes, a $10 \%$ wavespeed perturbation provides a rather satisfying explanation for the on-axis power deficit and off-path enhancement (left panel of Fig. 4).

\section{Conclusion}

We have measured the travel-time shifts and amplitudes of plane wave packets as they traverse a sunspot using a cross-covariance technique. Observations show on-axis wavefront healing and amplitude reduction. Travel-time anomalies spread in the transverse direction with distance and amplitude enhancements are seen away from the central ray. Wavefront healing is a finitewavelength phenomenon, which cannot be modeled by ray theory. To the best of our knowledge, the only other direct observational evidence of finite-wavelength effects in time-distance helioseismology is reported by Duvall et al. (2006), who studied the interaction of waves with subwavelength magnetic features.

We saw that ray tracing (unlike linearized ray theory) is quite useful for studying the geometrical attenuation caused by the defocusing of wave energy by a fast wave-speed anomaly. Physical wave absorption by the sunspot further enhances this reduction in the wave amplitude. The method of ray tracing is also useful for interpreting off-path amplitude enhancements.

The observations of travel-time shifts and wave amplitude that have been presented in this paper contain a wealth of information about the seismic signature of sunspots. Their interpretation requires more sophisticated methods of analysis than linearized ray theory (which is still often used in sunspot seismology today). A promising approach is to solve the wave equation numerically (Schunker et al. 2013, Paper II), in combination with iterative inversion methods (Hanasoge et al. 2011, 2012).

Acknowledgements. L.G. and H.S. acknowledge support from Deutsche Forschungsgemeinschaft SFB 963 "Astrophysical Flow Instabilities and Turbulence" (Project A18). Z.C.L. thanks the Max-Planck-Institut für Sonnensystemforschung for their hospitality. Z.C.L. was supported by the NSC of R.O.C. under the Study Abroad Program grant NSC98-2917-I-007-121. Partial support was also provided by ERC Starting Grant \#210949 "Seismic Imaging of the Solar Interior" to PI Gizon. The German Data Center for SDO, funded by the German Aerospace Center (DLR), provided the IT infrastructure required to carry out this work. SOHO is a project of international cooperation between ESA and NASA.

\section{References}

Braun, D. C., Duvall, Jr., T. L., \& Labonte, B. J. 1987, ApJ, 319, L27 Braun, D. C., Duvall, Jr., T. L., \& Labonte, B. J. 1988, ApJ, 335, 1015 Cally, P. S., \& Bogdan, T. J. 1997, ApJ, 486, L67

Cameron, R., Gizon, L., \& Duvall, Jr., T. L. 2008, Sol. Phys., 251, 291

Dahlen, F. A., \& Tromp, J. 1998, Theoretical Global Seismology (Princeton University Press)

Duvall, Jr., T. L., Birch, A. C., \& Gizon, L. 2006, ApJ, 646, 553

Gizon, L., \& Birch, A. C. 2002, ApJ, 571, 966

Gizon, L., Hanasoge, S. M., \& Birch, A. C. 2006, ApJ, 643, 549

Gizon, L., Schunker, H., Baldner, C. S., et al. 2009, Space Sci. Rev., 144, 249

Gizon, L., Birch, A. C., \& Spruit, H. C. 2010, ARA\&A, 48, 289

Hanasoge, S. M., Birch, A., Gizon, L., \& Tromp, J. 2011, ApJ, 738, 100

Hanasoge, S., Birch, A., Gizon, L., \& Tromp, J. 2012, Phys. Rev. Lett., 109, 101101

Gudmundsson, O. 1996, Geophys. J. Int., 124, 304

Hung, S., Dahlen, F. A., \& Nolet, G. 2001, Geophys. J. Int., 146, 289

Moradi, H., Baldner, C., Birch, A. C., et al. 2010, Sol. Phys., 267, 1

Nolet, G., \& Dahlen, F. A. 2000, J. Geophys. Res., 105, 19043

Scherrer, P. H., Bogart, R. S., Bush, R. I., et al. 1995, Sol. Phys., 162, 129

Schunker, H., Gizon, L., Cameron, R. H., \& Birch, A. C. 2013, A\&A, 558, A130 (Paper II)

Spruit, H. C., \& Bogdan, T. J. 1992, ApJ, 391, L109

Zaroli, C., Debayle, E., \& Sambridge, M. 2010, Geophys. J. Int., 182, 1025 